\title{
A prospective comparative study of microscope-integrated intraoperative fluorescein and indocyanine videoangiography for clip ligation of complex cerebral aneurysms
}

\author{
Brandon Lane, MD, Bradley N. Bohnstedt, MD, and Aaron A. Cohen-Gadol, MD, MSc \\ Goodman Campbell Brain and Spine, Department of Neurological Surgery, Indiana University, Indianapolis, Indiana
}

\begin{abstract}
OBJECT The authors prospectively analyzed 2 microscope-integrated videoangiography techniques using intravenous indocyanine green (ICG) and fluorescein for assessment of cerebral aneurysm obliteration and adjacent vessel patency. METHODS The authors prospectively enrolled 22 patients who underwent clip ligation of their aneurysm and used intraoperative videoangiography to assess obliteration of the aneurysmal sac and patency of the adjacent branching and perforating arteries. Patients underwent ICG videoangiography (ICG-VA) and the newly developed fluorescein videoangiography (FL-VA) using microscope-integrated fluorescence modules. Two independent observers compared the videoangiography recordings for value and quality to assess aneurysm exclusion and the patency of adjacent arteries.
\end{abstract}

RESULTS All 22 patients first underwent FL-VA and then ICG-VA after clip application. In 7 cases (32\%), FL-VA provided superior detail to assess perforating arteries (4 cases), distal branches (2 cases), and both (1 case); such detail was not readily available on ICG-VA. In 1 patient, ICG-VA offered better visualization of posterior communicating artery aneurysm occlusion than FL-VA because of staining artifact on the aneurysm dome from the adjacent tentorium. In 2 patients, FL-VA offered the needed advantage of real-time manipulation of the vessels and flow assessment by visualization through the operating microscope oculars. In 2 other cases, ICG-VA was more practical for repeat usage because of its more efficient clearance from the intravascular space. The ICG-VA image quality was often degraded at higher magnification in deep operative fields, partly due to chromatic aberration. Both ICG-VA and FL-VA afforded restricted views of vasculature based on the angle of surgical approach and obscuration by blood clot, aneurysm, or brain tissue.

CONCLUSIONS Compared with ICG-VA, FL-VA can potentially provide an improved visualization of vasculature at high magnification in deep surgical fields. ICG-VA is more effective for repeated use during clip repositioning due to ICG's minimal vascular wall extravasation. Therefore, in certain cases, FL-VA may offer some advantages and play a complementary role along with ICG-VA in intraoperative fluorescence evaluation during microsurgical management of aneurysms.

http://thejns.org/doi/abs/10.3171/2014.10.JNS132766

KEY WORDS fluorescein; indocyanine green; videoangiography; clip ligation; perforators; surgical technique

$\mathrm{T}$ HE use of fluorescent technologies in neurovascular imaging has been established with the use of indocyanine green (ICG) videoangiography in aneurysm surgery. The application of ICG was first reported in 2003 as a new method for qualitative intraoperative blood flow assessment. ${ }^{6}$ Although intraoperative digital subtraction angiography remains the gold standard to assess parent vessel patency and aneurysm obliteration, this method is time consuming, carries procedural risk, and may not assess small perforating vessels in a timely fashion. Intraoperative microvascular Doppler sonography is another tool for assessing patency of larger vessels and aneurysms; however, its utility is limited for evaluation of small perforating arteries. Therefore, intraoperative ICG fluorescence angiography is a surgical adjunct that provides a worthwhile tool for confirmation of aneurysm occlusion

ABBREVIATIONS ACOA = anterior communicating artery; FL-VA = fluorescein videoangiography; ICA = internal carotid artery; ICG = indocyanine green; ICG-VA = ICG videoangiography; $\mathrm{MCA}=$ middle cerebral artery; $\mathrm{PCOA}=$ posterior communicating artery; $\mathrm{PICA}=$ posterior inferior cerebellar artery; $\mathrm{SAH}=$ subarachnoid hemorrhage. ACCOMPANYING EDITORIAL See pp 616-617. DOI: 10.3171/2014.8.JNS14957.

SUBMITTED December 13, 2013. ACCEPTED October 27, 2014.

INCLUDE WHEN CITING Published online December 19, 2014; DOI: 10.3171/2014.10.JNS132766.

DISCLOSURE No funding was received for this study. The authors have no conflicts of interest in regard to this study except that Aaron A. Cohen-Gadol, MD, MSc, has a consulting agreement with Carl Zeiss Meditec, AG. The compensation from this arrangement is donated to a not-for-profit educational organization, The Neurosurgical Atlas. 
and the patency of branching and perforating vessels. This technology has been integrated into an operating microscope to allow for a practical, timely, and safe vascular flow evaluation. ${ }^{7}$

Indocyanine green is not the only fluorophore studied for intraoperative angiography. In vascular studies by Wrobel et al., ${ }^{11}$ perforating medium and large arteries were visualized following aneurysmal clip ligation using fluorescein angiography. In 2007, Suzuki et al. ${ }^{9}$ reported on the development of a pencil-type probe with a light-emitting diode to allow fluorescein fluorescence interrogation of the arteries deep inside the surgical field. These authors reported excellent image quality and spatial resolution.

In this study, we investigated the use of a recently developed microscope-integrated fluorescein videoangiography (FL-VA) fluorescent module and compared its imaging results to those of ICG videoangiography (ICG-VA) for management of complex aneurysms. These aneurysms were selected because of their location in relatively deep operative fields and/or incorporation of perforating or branching arteries within their wide neck. In these situations, intraoperative flow assessment is important to prevent cerebral infarction and confirm effective aneurysm exclusion. Similar to ICG-VA, FL-VA is a microscope-integrated module that allows for a reduction in the required dose of intravascular fluorescein compared with doses used in prior studies, while maintaining adequate vascular fluorescence imaging. More importantly, this technology allows the operator to visualize the surgical field in real time through the operating oculars under the fluorescent mode while identifying the vessels of interest.

\section{Methods \\ Patient Population}

This study included 22 prospectively enrolled patients who underwent clip ligation of their intracranial aneurysms during November 2012 through November 2013 using both FL-VA and ICG-VA (Table 1). These 22 patients were included because their aneurysms were located in a relatively narrow deep operative corridor (we have previously encountered suboptimal fluorescence imaging in these situations using ICG-VA) or the broad neck of their aneurysms incorporated adjacent branching and perforating arteries. There were 21 female patients and 1 male patient who ranged in age from 24 to 69 years (mean 47 years). All patients underwent standard microsurgical procedures for clip ligation of their aneurysms followed by FL-VA and ICG-VA. The Indiana University Institutional Review Board reviewed and approved this study, and all patients provided consent before participation.

The chi-square test was used to compare the superiority of FL-VA versus ICG-VA in evaluating small perforators, branching arteries, and aneurysmal obliteration, and in their ability to provide useful information on repeat imaging. These parameters were analyzed together to allow for enough patients for meaningful statistical analysis.

\section{Sodium Fluorescein/ICG and Their Intraoperative Fluorescence Modules}

We used an OPMI PENTERO 900 neurosurgical oper- ating microscope with a YELLOW 560 integrated fluorescence filter module for imaging fluorescein fluorescence, and we used an integrated fluorescence-based videoangiography camera module (INFRARED 800) for visualizing ICG fluorescence (Carl Zeiss Meditec, AG). The YELLOW 560 module delineates the fluorescent signal using intravascular sodium fluorescein and visualizes nonfluorescent tissue in a relatively dim background (based on the depth of the operative field), where manipulation of vessels is often safe and possible. This module is optimized for excitation in the range of 460-500 $\mathrm{nm}$ and an emission range between 540 and $690 \mathrm{~nm}$, which matches those of sodium fluorescein. For generating a white light impression of nonfluorescent tissue, an optical mixing of light using specifically defined amounts of blue and red light are created. This technology allows improved observation of tissue details and often optimizes intraoperative vascular manipulation under the fluorescence mode using the operator's microscope oculars.

The INFRARED 800 fluorescence videoangiography camera separates emission and excitation light, so only fluorescent areas are visible, but nonfluorescent areas appear black. The INFRARED 800 is designed for an excitation range between 700 and $780 \mathrm{~nm}$ and emission detection in the range of 820 and $900 \mathrm{~nm}$. The ICG signal emission cannot be seen through the operating oculars, and it must be processed and displayed on a monitor as it is formed in the infrared region of the electromagnetic spectrum. Therefore, presently, only fluorescein fluorescence allows visualization through the operator's oculars and allows manipulation of branching and perforating arteries to inspect their flow status under the fluorescence mode. Manipulation of vessels under the fluorescent mode is possible using the ICG module as long as the assistant reports the findings to the surgeon.

For each patient, both ICG-VA and FL-VA were done at the same microscope's focal length and magnification level. The magnification was set at a level to adequately visualize the vascular anatomy and relevant perforating and branching arteries. The images for both FL-VA and ICGVA were digitally recorded on a storage device and later reviewed for this analysis.

\section{Results}

\section{Sodium Fluorescein Videoangiography}

After aneurysm clip ligation, we administered a 75$\mathrm{mg}$ bolus intravenous dose of sodium fluorescein (Akorn, Inc.) through a peripheral intravenous line, and the intracranial area of interest was inspected through the microscope integrated YELLOW 560 module. Approximately 20 seconds later, cerebral arterial, capillary, and venous phases were observed fluorescing through the oculars in yellow-green colors. In our experience, this is the lowest dose that maintains adequate fluorescent signal detectable within the arteries using the YELLOW 560 module. We determined this dose based on a preliminary study of 8 patients, during which we used gradually declining doses at 25-mg increments, starting from $200 \mathrm{mg}$, for imaging.

We were able to visualize the fluorescent vessels through the operating oculars and investigate fluorescence within the surrounding vessels. Real-time manipulation 


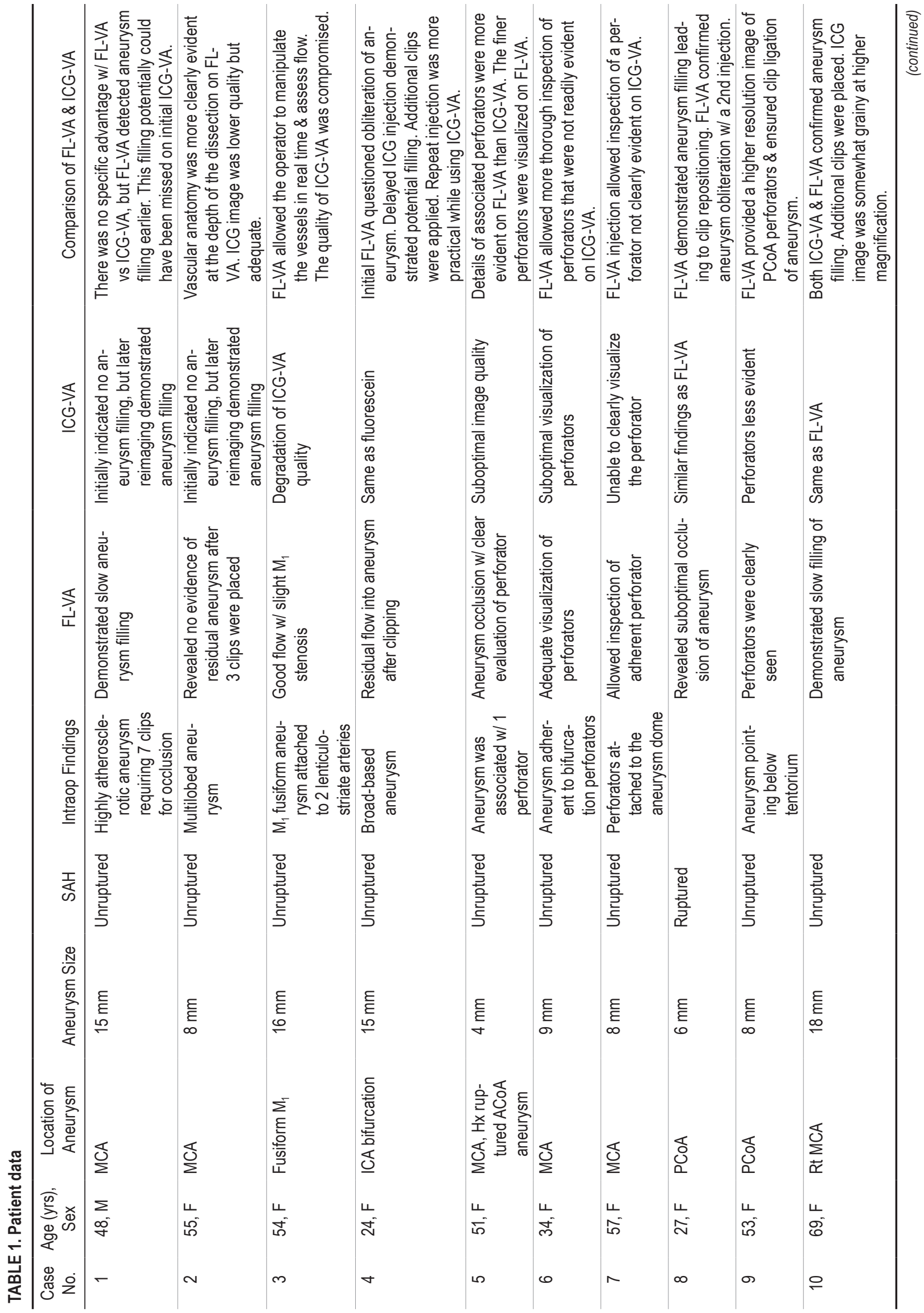




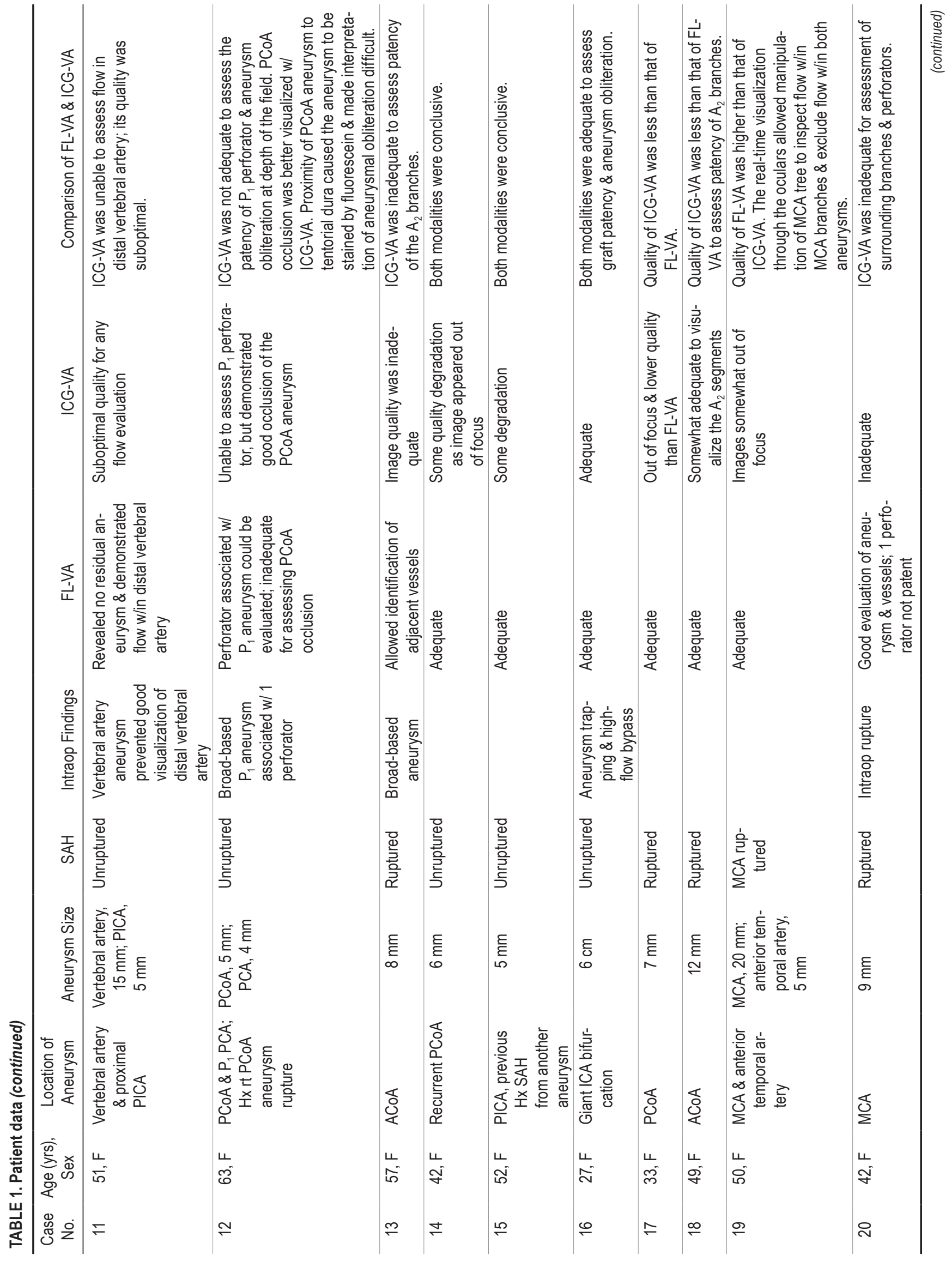


of parent vessels, branching vessels, and perforators was possible to ensure their patency. The light intensity was relatively dim within the deep operative fields (posterior circulation aneurysms) compared with those under the conventional white light mode. Due to its porous vascularity, the dura was stained with fluorescent signal. After 20-30 minutes, the vessels cleared most of the fluorescent signal. A minor amount of fluorescence was also evident in the CSF after 10 minutes. Although redosing was used in 2 cases to investigate flow after clip repositioning, these aneurysms did stain somewhat with the fluorescein from the initial injection, making reimaging interpretation difficult. Importantly, our patients did not experience any complication from the use of sodium fluorescein.

\section{Indocyanine Green Videongiography}

Following FL-VA, all patients underwent ICG-VA (25$\mathrm{mg}$ intravenous bolus) for evaluation of vasculature and aneurysm. The INFRARED 800 module was used to assess blood flow dynamics by examining the images on an adjacent monitor. This method did not allow the surgeon to dissect or manipulate the parent, branching, or perforating vessels under the fluorescence mode in real time. Reinjection of the dye after 10 minutes for patients who underwent clip repositioning allowed reasonable interpretation of imaging, even though some mild fluorescence was still present from the initial injection.

\section{FL-VA and ICG-VA Comparison Data}

Twenty-two patients, each with at least 1 cerebral aneurysm, underwent fluorescent videoangiography, including first FL-VA and then ICG-VA after clip application. The average aneurysm size was $16 \mathrm{~mm}$ (range 4-60 mm). Seven aneurysms were ruptured and 18 were unruptured. Of these 22 patients, 3 underwent concurrent clip ligation of 2 aneurysms through the same pterional approach. All but 2 patients underwent clip ligation of their saccular aneurysms. Regarding these 2 patients, 1 patient harbored a giant internal carotid artery (ICA) bifurcation aneurysm and underwent ICA bifurcation-radial artery-middle cerebral artery (MCA) revascularization followed by aneurysm trapping through clip ligation of the ICA and $\mathrm{A}_{1}$ segment. The second patient underwent superficial temporal arteryto-MCA bypass followed by trapping of a giant fusiform $\mathrm{M}_{2}$ aneurysm.

In 7 patients (32\%), FL-VA provided superior detail to allow adequate assessment of perforating arteries (4 cases) or distal branches (2 cases) or both (1 case); such detail was not readily available on ICG-VA. The superiority of FL-VA image quality was evident in 4 patients $(18 \%)$ for assessing ineffective aneurysm obliteration (1 case) or potentially avoiding intraoperative angiography (3 cases). In these 3 patients (Cases 12, 13, and 18 [Table 1]), the quality of ICGVA was suboptimal. When compared with ICG-VA, FLVA did not affect clip repositioning in any of our patients, which was performed to avoid injury to corresponding perforators. Seven other patients underwent intraoperative arteriography after clip ligation and fluorescence imaging of their vessels and aneurysms due to restricted views of these structures using ICG-VA and FL-VA techniques. When 
comparing all of the study parameters, including the ability to evaluate small perforators, branching arteries, aneurysmal obliteration, and useful information on repeat imaging, FL-VA was superior to ICG-VA $\left(\chi^{2}=7.111, p=0.008\right)$.

In 1 patient (Case 12, Table 1), ICG-VA offered better visualization of posterior communicating artery (PCoA) aneurysm occlusion than FL-VA because of staining artifact of the aneurysm dome due to extravasation of dye from the adjacent tentorium. In 2 patients (Cases 3 and 19, Table 1), FL-VA offered the needed advantage of real-time manipulation (visualization under the fluorescent mode through the oculars) of the vessels to expose the vessels and aneurysms of interest. In Case 3, the perforating arteries were hidden behind the clip blades, and clip manipulation and aneurysm mobilization were necessary for their visualization. In Case 19, there were 2 adjacent aneurysms, and manipulation of $\mathbf{M}_{1}$ was necessary to detect both aneurysms and ensure their obliteration. In 2 patients (Cases 4 and 12, Table 1), ICG-VA was more practical for repeat usage because of its more efficient clearance from the intravascular space. The ICG-VA image quality was often degraded at higher magnification in deep operative fields, partly due to the phenomenon of chromatic aberration. Both ICG-VA and FL-VA afforded restricted views of vasculature based on the angle of surgical approach and obscuration by blood clot, aneurysm, or brain tissue. Overall, FL-VA was useful throughout the magnification range of the microscope and allowed for better imaging of small and perforating arteries among deep-seated aneurysms.

In 1 patient (5\%) (Case 1, Table 1 ), the FL-VA demonstrated aneurysmal filling while ICG-VA found the opposite. This aneurysm was highly atherosclerotic and calcified (see Case 1 below for further details). Two patients (9\%) suffered from infarcts related to perforating vessel injury on postoperative imaging, one of whom was temporarily symptomatic. One of these infarcts was due to an injury to a PCoA perforator and was approach related and not due to clip application (Case 12, Table 1). The other perforator sacrifice was related to clip application on a fusiform aneurysm (Case 3, Table 1) and was evident on intraoperative FL-VA and not ICG-VA. Despite various maneuvers, this perforator could not be salvaged.

\section{Illustrative Cases}

\section{Case 11}

A 51-year-old woman with an incidental 15-mm left vertebral artery aneurysm and a 5-mm adjacent posterior inferior cerebellar artery (PICA) aneurysm underwent a left suboccipital craniotomy and clip ligation of both aneurysms (Table 1). Because of the depth of the field and the potential lack of an excitation signal adequately illuminating the aneurysm, the ICG images were suboptimal, and we were unable to adequately assess the flow in the distal vertebral artery and the PICA. FL-VA demonstrated a patent vertebral artery and aneurysm obliteration (Fig. 1).

\section{Case 1}

A 48-year-old man presented with an unruptured 15mm right MCA aneurysm. After aneurysm clip ligation, FL-VA demonstrated aneurysm filling (Table 1). The first attempt using ICG-VA did not demonstrate this finding, but reimaging with ICG-VA a few minutes later confirmed the FL-VA findings. This discrepancy could be due to the significantly atherosclerotic wall of the aneurysm, which prevents the larger ICG molecule from penetrating and fluorescing through the aneurysm wall (Fig. 2).

\section{Case 12}

This 63-year-old woman with a history of subarachnoid hemorrhage (SAH) from a right PCoA aneurysm managed endovascularly also harbored an incidental 5-mm broadbased right $\mathrm{P}_{1}$ posterior cerebral artery aneurysm and a 6-mm left PCoA aneurysm (Table 1). Through a left pterional approach, we treated the contralateral $\mathrm{P}_{1}$ aneurysm with clip ligation and found ICG-VA inadequate to assess the patency of the small associated perforator and aneurysm obliteration. Using FL-VA, we were able to overcome these shortcomings.

The occlusion of the PCoA aneurysm was better visualized using ICG. The proximity of the PCoA aneurysm to the tentorial dura led to staining of the aneurysm dome by fluorescein (due to the first fluorescein injection to evaluate the $\mathrm{P}_{1}$ aneurysm) and made interpretation of aneurysmal obliteration difficult (Fig. 3).

\section{Case 7}

A 57-year-old woman presented with an 8-mm left MCA aneurysm (Table 1). This aneurysm was clip ligated through a pterional approach. Although FL-VA demonstrated patency of adjacent perforators, ICG-VA under the same magnification did not provide similar data (Fig. 4).

\section{Case 13}

This 57-year-old woman presented with a ruptured 8-mm anterior communicating artery (ACoA) aneurysm (Table 1). She underwent clip ligation through a left-sided pterional craniotomy. The patency of the $\mathrm{A}_{2}$ branches was more evident on FL-VA compared with ICG-VA (Fig. 5).

\section{Discussion}

Fluorescence videoangiography provides a safe and practical imaging modality to intraoperatively assess blood flow dynamics within aneurysms and adjacent vessels without interfering with surgical workflow. ICG and fluorescein are 2 unique fluorophores used successfully as surgical adjuncts in aneurysm surgery, and, based on their unique chemical properties, they provide distinct advantages and disadvantages..$^{9,11}$ At least 3 other groups of investigators have reported the drawbacks of ICG-VA related to its poor image quality in deep operative fields. ${ }^{1-3,10}$ More specifically, these investigators found a higher incidence of discordance between ICG-VA and postoperative angiograms during ACoA aneurysm surgery. ${ }^{1,2,10}$ They considered the narrow field of view and anatomical complexity of the region as potential causes for these drawbacks.

The advantages of ICG-VA include its more widespread use since its introduction for cerebrovascular surgery and the ability to perform repeated injections within minutes for flow reevaluation after clip repositioning. Based on our study, the advantages of FL-VA include its improved reso- 

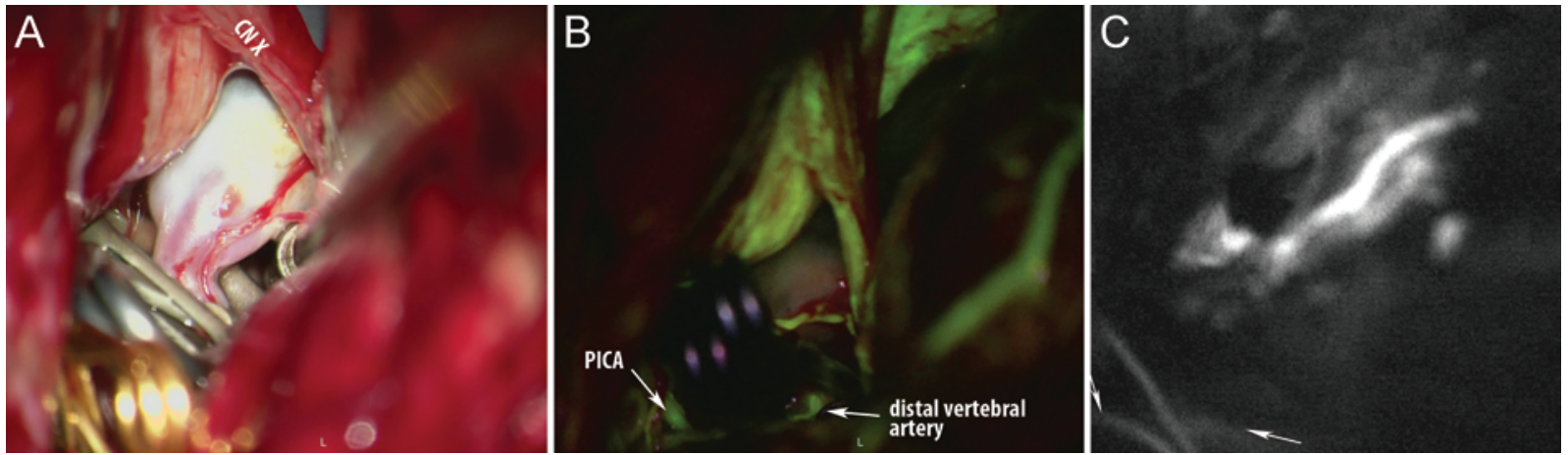

FIG. 1. Case 11. A 51-year-old woman presented with an incidental $15-\mathrm{mm}$ left vertebral artery and a 5-mm adjacent PICA aneurysm. A fenestrated straight clip and a simple straight clip were placed to exclude the vertebral artery aneurysm (CN X $=10$ th cranial nerve) (A). FL-VA demonstrated patent vertebral artery and aneurysm obliteration (B). Due to the depth of field and potential lack of excitation signal adequately illuminating the aneurysm, ICG images at similar magnification were suboptimal, and we were unable to adequately assess the flow in the distal vertebral artery and the PICA (C). Figure is available in color online only.

lution to detect flow within branching and small perforating arteries at deep operative corridors that need to be examined under high magnification. Such operative corridors often may require the surgeon to mobilize the surrounding structures and vessels to see the fluorescence signal in real time through the operating oculars within vessels of interest. This ability is especially important if the difference between retrograde flow within a vessel (due to proximal stenosis by the clip blades) versus an anterograde flow can be appreciated in real time. In the past, there have been concerns about patchy fluorescent staining of vessels, but this was restricted to large parent arteries and was not observed among medium and small perforating arteries. ${ }^{8}$

Fluorescein fluorescence may also be used under circumstances in which initial ICG-VA reveals a lack of an- eurysm exclusion and clip repositioning requires reimaging. In this situation, the operator can use FL-VA for reimaging and does not need to wait for complete clearance of the initial dose of ICG. Therefore, we recommend the use of FL-VA as a complementary tool in addition to ICG-VA for intraoperative vascular imaging.

\section{Chromatic Aberration}

During our study, depth of field and high magnification impacted ICG-VA image quality more significantly based on principles of chromatic aberration. The phenomenon of chromatic aberration explains why the light arrays of different wavelengths own different focal points. ${ }^{4}$ This discrepancy in focal point can be compensated for, to some extent, by using apochromatic optics. Apochromatic optics
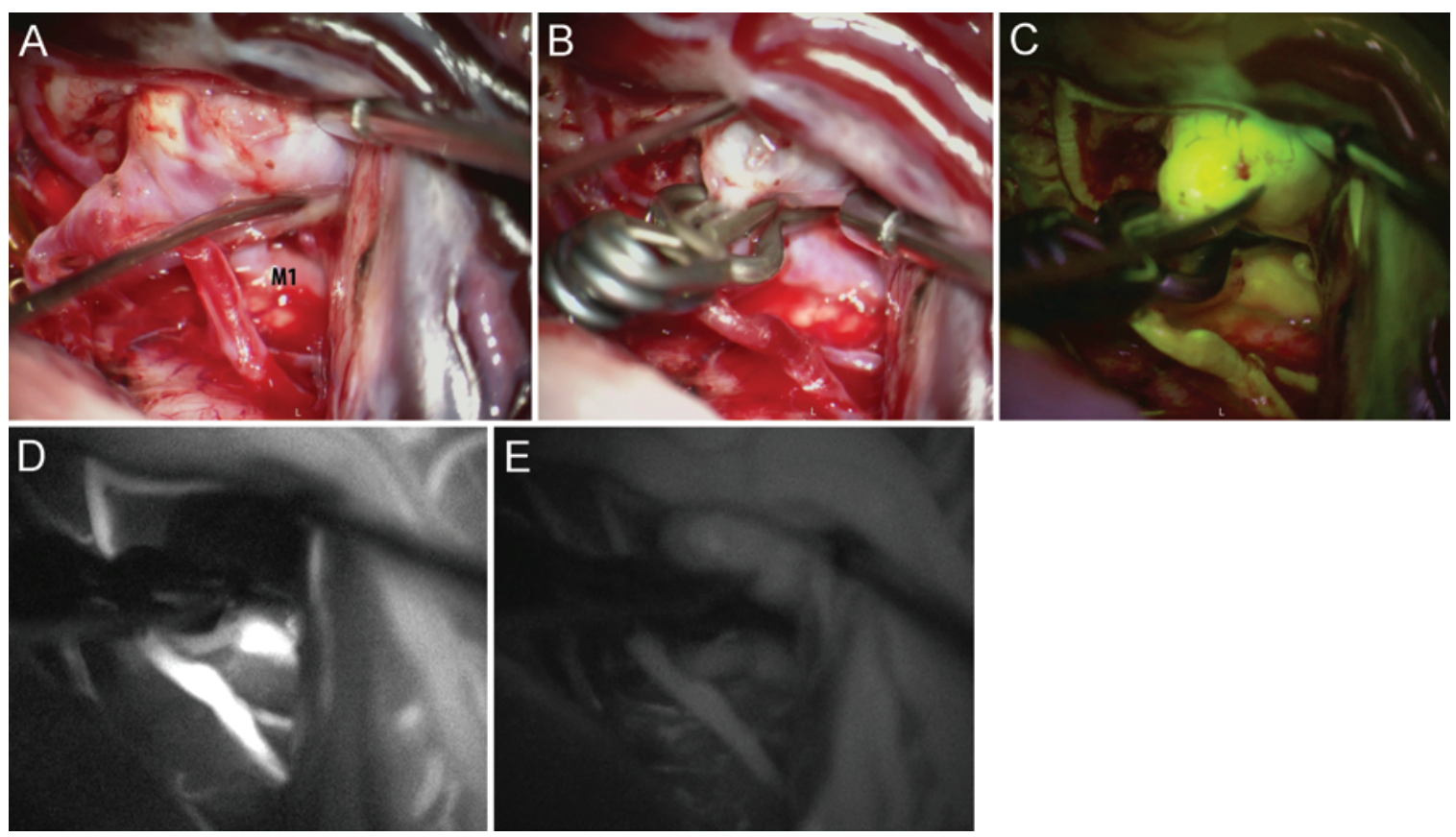

FIG. 2. Case 1. A 48-year-old man presented with an unruptured 15-mm atherosclerotic right MCA aneurysm (A). After aneurysm clip ligation (B), FL-VA demonstrated aneurysm filling (C). The first attempt using ICG-VA did not demonstrate this finding (D), but reimaging a few minutes later confirmed the FL-VA findings, although the image was degraded (E). Following placement of additional clips, we confirmed aneurysm exclusion by puncturing the dome. Figure is available in color online only. 

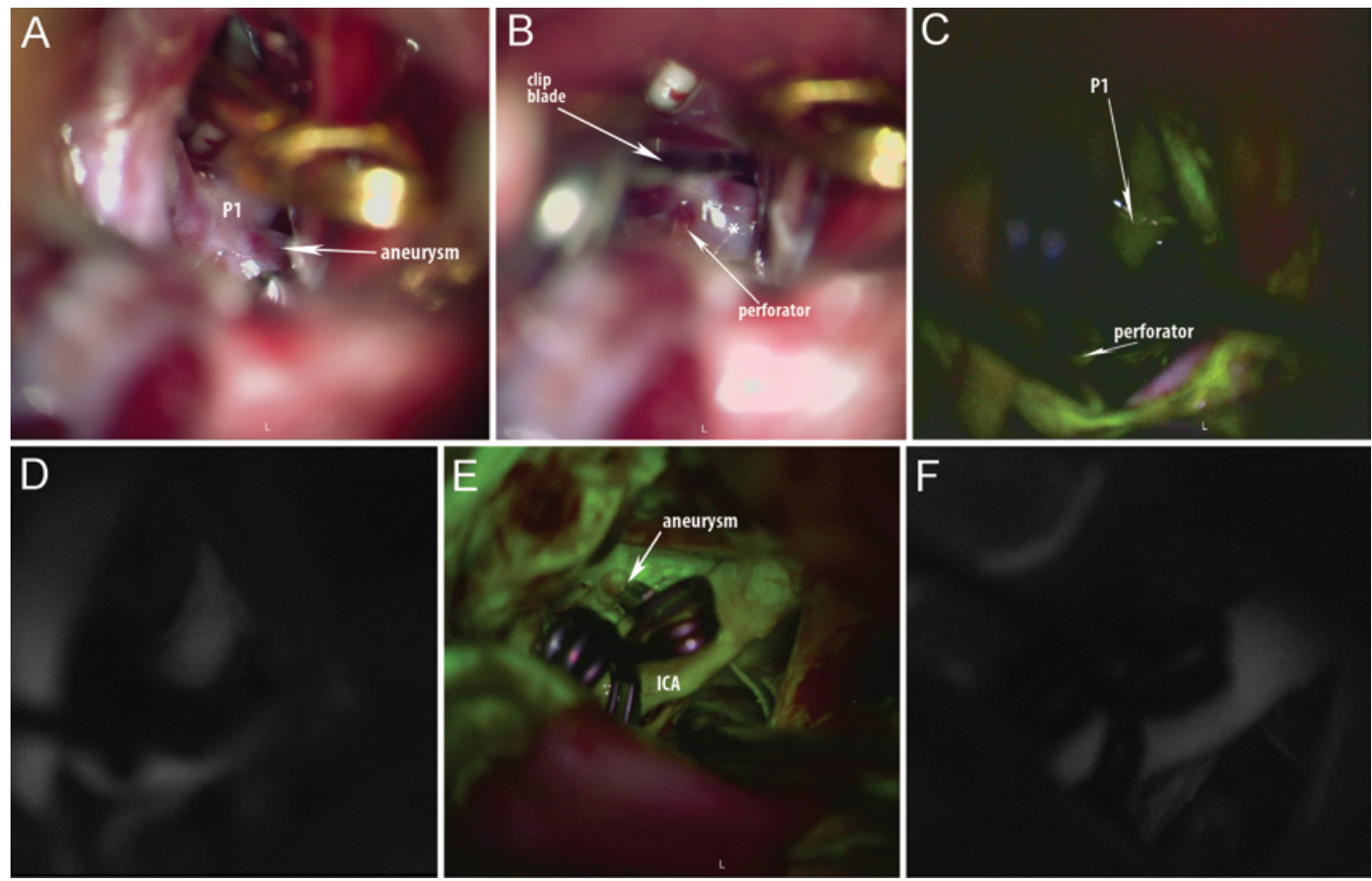

FIG. 3. Case 12. This 63-year-old woman with a previous history of SAH from a right PCoA aneurysm harbored an incidental 5-mm broad-based right $P_{1}$ aneurysm $(A)$ and a 6-mm left $P C o A$ aneurysm. Through a left pterional approach, we reached the $P$ aneurysm through a "cross-court" route and ligated it with a clip (B). With FL-VA, we were able to confirm aneurysm obliteration, patency of $\mathrm{P}_{1}$, and, most importantly, the associated perforator (C). ICG-VA was inadequate to assess the patency of the small associated perforator and aneurysm obliteration (D). The proximity of the PCoA aneurysm to the tentorial dura led to staining of the aneurysm dome by fluorescein (due to the first fluorescein injection to evaluate the $P_{1}$ aneurysm) and made interpretation of aneurysmal obliteration difficult $(\mathrm{E})$. The occlusion of the PCoA aneurysm was better visualized using ICG (F). Figure is available in color online only.

use special lenses that help focus lights of different wavelengths on the same plane. This technique is most beneficial for corrections in the visible light spectrum (400-700 $\mathrm{nm})$. Notably, the emission light for ICG $(820-900 \mathrm{~nm})$ falls outside the visible but within the near-infrared spectrum. Light in this range can still be compensated for in certain instances.

There is less discrepancy among the focal points at lower magnifications and in a field about $300 \mathrm{~mm}$ deep. This is partly the reason for the recommended setting of 300 $\mathrm{nm}$ and $5 \times$ zoom during the use of an INFRARED 800 module. At higher magnifications, the focus shift between near-infrared light and white light becomes more criti- cal, with resultant blurry and degraded ICG-VA images. The limited reach of the excitation light within these deep corridors further compounds generation of an adequately detectable emission ICG fluorescent signal. These limitations affect FL-VA less dramatically because fluorescein's excitation and emission peaks, 465-490 and 520-530, respectively, both fall within the visible spectrum where apochromatic compensation is optimal.

\section{Other Considerations}

When compared with ICG, fluorescein leaks more readily into the extravascular space and into the aneurysm wall within a few minutes after injection. This leads to a need
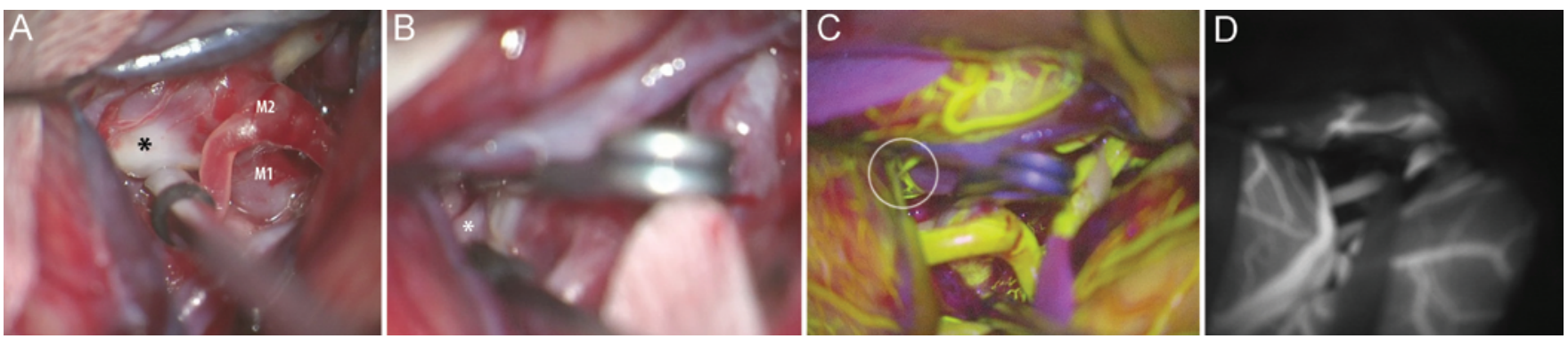

FIG. 4. Case 7. A 57-year-old woman presented with an unruptured 8-mm left MCA bifurcation aneurysm (asterisk) (A). This aneurysm was treated using clip ligation through a pterional approach using a straight clip (B). Although FL-VA demonstrated patency of the adjacent perforators (circled) (C), ICG-VA under the same magnification was unable to provide similar data (D). Figure is available in color online only. 

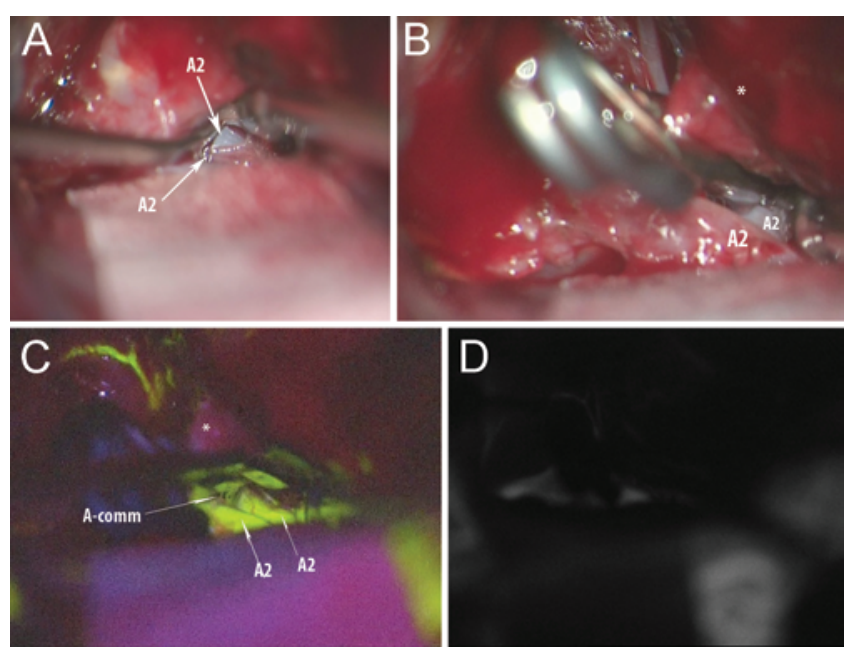

FIG. 5. Case 13. This 57-year-old woman presented with a ruptured 8-mm ACoA aneurysm (A). She underwent clip ligation through a leftsided pterional craniotomy (B). The patency of the $A_{2}$ branches was more evident on FL-VA (C) compared with ICG-VA (D). Figure is available in color online only.

for a longer period of time for fluorescein fluorescence to clear to allow for a repeated injection to assess the result of clip repositioning. Given this effect, there is a challenge with repeated administration of fluorescein. Similarly, fluorescein has a stronger proclivity to stain dural membranes and adjacent aneurysm domes if it leaks extravascularly. As we mentioned in our discussion of Case 12 (Table 1), assessment of the patency of adjacent perforators or aneurysm sac obliteration can be difficult because of adjacent dural leakage and staining of neighboring vascular structures. This can be a problem with repeated injections for PCoA aneurysms because of the proximity of the tentorium. Most importantly, in lower doses, as we have discussed in this article, fluorescein most likely has a safety profile comparable to that of ICG. The dose used in this study was $15 \%$ (75 mg compared with $500 \mathrm{mg}$ used previously) of the dose that has been used in ophthalmology applications.

Kuroda et al. ${ }^{5}$ recently reported on the use of intraarterial fluorescein and the advantage of this technique. Particularly, they demonstrated brighter and sharper fluorescence contrast with a smaller dose of dye, faster passage (which allowed shorter repetition intervals without residual staining), and assessment of the exact stream of blood flow in the parent and perforating arteries. This technique may facilitate multiple FL-VA studies with minimal residual staining. Therefore, in patients who already harbor an intraarterial catheter, this method might further increase the utility of fluorescein fluorescence in aneurysm surgery.

\section{Conclusions}

During both ICG and fluorescein videoangiography, imaging is restricted to the angle of the surgical approach and exposed vasculature. Vessels covered by blood clots, aneurysm, or brain tissue are not visible using these techniques. On the other hand, both of these modalities are easily performed and are potentially complementary. ICG is optimal for repeat usage and remains strictly intravas- cular. ICG fluorescence image quality is limited at higher magnification within deep operative fields. On the other hand, fluorescein fluorescence can be used with a full range of magnification and can be visualized in real time through the operating oculars within deep surgical fields to better image smaller vessels such as perforators.

\section{References}

1. Dashti R, Laakso A, Niemelä M, Porras M, Hernesniemi J: Microscope integrated indocyanine green video-angiography in cerebrovascular surgery. Acta Neurochir Suppl 109:247250, 2011

2. Dashti R, Laakso A, Niemelä M, Porras M, Hernesniemi J: Microscope-integrated near-infrared indocyanine green videoangiography during surgery of intracranial aneurysms: the Helsinki experience. Surg Neurol 71:543-550, 2009

3. Gruber A, Dorfer C, Standhardt H, Bavinzski G, Knosp E: Prospective comparison of intraoperative vascular monitoring technologies during cerebral aneurysm surgery. Neurosurgery 68:657-673, 2011

4. ISO 19012-2:2009. Optics and photonics-designation of microscope objectives-Part 2: Chromatic correction. International Organization for Standardization. (http://www. iso.org/iso/iso_catalogue/catalogue_ics/catalogue_detail_ics. htm?csnumber=46241) [Accessed October 28, 2014]

5. Kuroda K, Kinouchi H, Kanemaru K, Nishiyama Y, Ogiwara $\mathrm{M}$, Yoshioka $\mathrm{H}$, et al: Intra-arterial injection fluorescein videoangiography in aneurysm surgery. Neurosurgery 72 (2 Suppl Operative):ons141-150, 2013

6. Raabe A, Beck J, Gerlach R, Zimmermann M, Seifert V: Near-infrared indocyanine green video angiography: a new method for intraoperative assessment of vascular flow. Neurosurgery 52:132-139, 2003

7. Raabe A, Nakaji P, Beck J, Kim LJ, Hsu FP, Kamerman JD, et al: Prospective evaluation of surgical microscopeintegrated intraoperative near-infrared indocyanine green videoangiography during aneurysm surgery. J Neurosurg 103:982-989, 2005

8. Raabe A, Spetzler RF: Fluorescence angiography. J Neurosurg 108:429-430, 2008 (Letter)

9. Suzuki K, Kodama N, Sasaki T, Matsumoto M, Ichikawa T, Munakata R, et al: Confirmation of blood flow in perforating arteries using fluorescein cerebral angiography during aneurysm surgery. J Neurosurg 107:68-73, 2007

10. Washington CW, Zipfel GJ, Chicoine MR, Derdeyn CP, Rich $\mathrm{KM}$, Moran CJ, et al: Comparing indocyanine green videoangiography to the gold standard of intraoperative digital subtraction angiography used in aneurysm surgery. J Neurosurg 118:420-427, 2013

11. Wrobel CJ, Meltzer H, Lamond R, Alksne JF: Intraoperative assessment of aneurysm clip placement by intravenous fluorescein angiography. Neurosurgery 35:970-973, 1994

\section{Author Correspondence}

Conception and design: all authors. Acquisition of data: all authors. Analysis and interpretation of data: all authors. Drafting the article: all authors. Critically revising the article: all authors. Reviewed submitted version of manuscript: all authors. Approved the final version of the manuscript on behalf of all authors: Cohen-Gadol. Study supervision: Cohen-Gadol.

\section{Correspondence}

Aaron A. Cohen-Gadol, Goodman Campbell Brain and Spine, Indiana University Department of Neurosurgery, 355 W. 16th St., Ste. 5100, Indianapolis, IN 46202. email: acohenmd@gmail.com. 